\section{$\underset{\substack{\text { hommes } \\ \text { \& migrations }}}{ }$}

\section{Hommes \& migrations}

Revue française de référence sur les dynamiques

migratoires

\section{8 | 2014}

Les Paris des migrants

\title{
Les Japonais à Paris
}

Entre invisibilité résidentielle et hypervisibilité commerciale

\section{Hadrien Dubucs}

\section{(2) OpenEdition}

12 Journals

\section{Édition électronique}

URL : http://journals.openedition.org/hommesmigrations/2992

DOI : 10.4000/hommesmigrations.2992

ISSN : 2262-3353

Éditeur

Musée national de l'histoire de l'immigration

\section{Édition imprimée}

Date de publication : 1 octobre 2014

Pagination : 45-52

ISBN : 978-2-919040-29-2

ISSN : 1142-852X

\section{Référence électronique}

Hadrien Dubucs, «Les Japonais à Paris », Hommes \& migrations [En ligne], 1308 | 2014, mis en ligne le 01 octobre 2017, consulté le 01 mai 2019. URL : http://journals.openedition.org/

hommesmigrations/2992 ; DOI : 10.4000/hommesmigrations.2992 


\title{
LES JAPONAIS À PARIS ENTRE INVISIBILITÉ RÉSIDENTIELLE ET HYPERVISIBILITÉ COMMERCIALE
}

par HADRIEN DUBUCS, géographe, maître de conférences à l'UFR de géographie et d'aménagement de l'université Paris-IV-Sorbonne, laboratoire Espace, nature et culture (UMR ENeC 8185).

\author{
Les migrants japonais à Paris, malgré la diversité \\ de leur situation sociale et professionnelle, peuvent compter \\ sur un système bien structuré d'acteurs et de ressources \\ qui concourent à créer une relative concentration résidentielle \\ dans le centre-ouest de Paris. La présence de ce groupe \\ relativement peu nombreux devient visible par une offre \\ commerciale croissante et en recomposition, historiquement \\ implantée dans le quartier de l'Opéra. Pour autant, il demeure \\ difficile de parler d'un quartier japonais à Paris.
}

Parmi les populations immigrées à Paris, les Japonais constituent à bien des égards un cas atypique : ils sont originaires de l'un des pays les plus riches du monde, alors que $45 \%$ des immigrés en Île-deFrance viennent d'Afrique; ils sont plus souvent diplômés du supérieur que l'ensemble des immigrés franciliens (60\% contre $30 \%$ ); parmi les seuls actifs, plus de $35 \%$ appartiennent à la catégorie "cadres et professions intellectuelles supérieures" contre environ $10 \%$ pour l'ensemble des immigrés ${ }^{1}$. Les caractéristiques symboliques du pays d’origine combinées avec les caractéristiques socioprofessionnelles des migrants tendent à faire des migrants japonais à Paris un exemple d'“élite" migrante ${ }^{2}$. L'accroissement du niveau moyen de qualification des immigrés en France, les restrictions faites à l'encontre de la migration de travail peu qualifié, la montée en puissance des mobilités internationales d'étudiants et de salariés au sein des firmes multinationales ont nourri depuis les années 1990 un nombre croissant de travaux sur les migrants internationaux occupant des positions socioprofessionnelles moyennes et élevées. Cependant, ces travaux mettent souvent davantage l'accent sur les évolutions quantitatives et les modalités de la migration elle-même que sur la territorialisation des individus dans les villes de séjour. Or c'est bien l'échelle fine des espaces urbains vécus et habités qui permet d'analyser comment les migrants internationaux, en fonction de capitaux sociaux 
et symboliques propres, peuvent contribuer à la production des villes qu'ils pratiquent. C'est cette perspective qui est abordée ici à partir d'un matériau empirique essentiellement qualitatif collecté depuis 2005 et qui porte sur la manière dont les Japonais résidant à Paris agencent leurs pratiques dans l'espace parisien.

\section{Paris, une place à part dans le champ migratoire japonais}

Par rapport à Londres et à Düsseldorf, autres principales villes de l'immigration japonaise en Europe, Paris se singularise par une grande diversité socioprofessionnelle parmi ses 10 à 20000 résidents japonais ${ }^{3}$. Les salariés expatriés et leurs familles n'en sont qu'une composante limitée (30 \% environ) et relativement tardive au regard d'une histoire migratoire qui a commencé dans les années 1920 par l'installation d'artistes et d'intellectuels. Cette tradition se perpétue aujourd'hui avec environ 2500 entrées d'étudiants japonais par an depuis les années 1990, dans des domaines où Paris a une forte renommée internationale : la gastronomie, la mode, l'artisanat d'art ou encore la musique.

Trois groupes socioprofessionnels se distinguent. Il s'agit premièrement de salariés expatriés au sein d'entreprises ou d'administrations japonaises, le plus souvent des hommes accompagnés de leurs épouse et enfants et qui séjournent deux à cinq ans à Paris. Le deuxième groupe est composé d'étudiants ou de stagiaires qui bénéficient d'un visa d'un an renouvelable sans trop de difficultés, et qui dans certains cas prolongent indéfiniment leur séjour au gré des opportunités professionnelles. Enfin, on relève un ensemble très hétérogène de migrants d'âge actif, majoritairement des femmes, aux parcours et positions socioprofessionnelles extrêmement variés. On y retrouve les professionnels indépendants dans le domaine artistique, mais aussi des diplômés occupant des postes précaires et peu qualifiés dans des sociétés ou des restaurants japonais.

La plupart du temps, le séjour à Paris répond à un projet de changement personnel ou professionnel qui peut se traduire par des formes au moins temporaires de déclassement. À l'instar des Britanniques immigrés à Paris ${ }^{4}$, les Japonais illustrent le cas d'une population globalement très qualifiée, mais au sein de laquelle la diversité des positions et plus encore des parcours socioprofessionnels ne saurait se réduire à la figure "élitaire" de l'expatrié ${ }^{5}$. Les résidents japonais de Paris s'apparentent plutôt à une classe moyenne aux contours socioprofessionnels difficiles à préciser, pour laquelle le séjour parisien revêt des significations très diverses, depuis la brève parenthèse estudiantine jusqu'au lieu d'ancrage professionnel et familial. La combinaison entre le projet associé à Paris et les ressources sociales et financières mobilisables induit une grande diversité des lieux pratiqués et habités.

\section{Des localisations résidentielles entre concentration et diversification}

La carte de la distribution résidentielle des Japonais dans l'espace parisien (figure 1) montre clairement le poids de Paris (60 \%) et, en deuxième lieu, celui des Hauts-de-Seine (20 \%) parmi l'ensemble des immigrés japonais dans l'espace francilien, qui concentre $70 \%$ des immigrés japonais en France ${ }^{6}$. Les XV et XVI ${ }^{e}$ arrondissements ainsi que Boulogne-Billancourt rassemblent la majorité des immigrés japonais.

Cette distribution relativement typée dans l'espace parisien s'explique d'abord par les modalités de l'accès au logement. Quel que soit leur profil socio- 
Figure 1. Localisations résidentielles en île-de-France des immigrés japonais (1999).

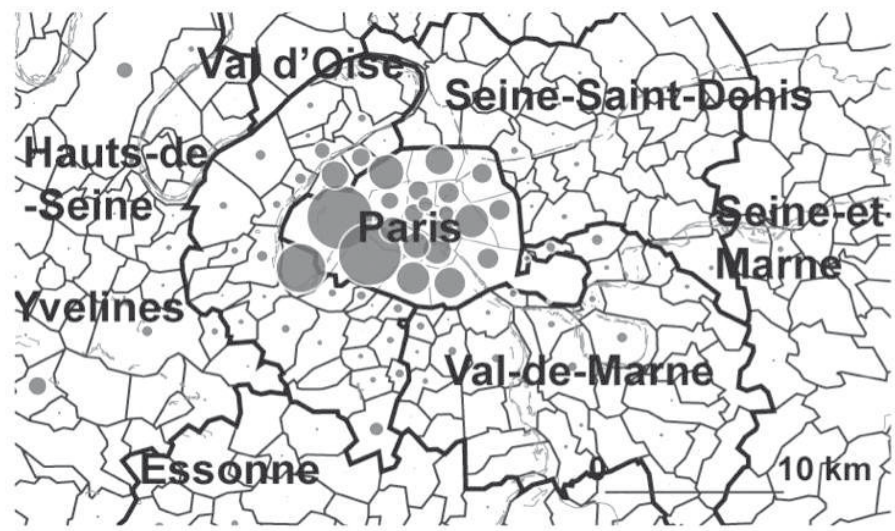

Immigrés japonais

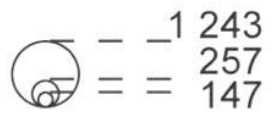

Réalisé avec Philcarto - http://perso.club-internet.fr/Philgeo.

Source : Insee, RGP, 1999. Champ : communes de plus

de 5000 habitants; population de 15 ans et plus.

professionnel, les immigrés japonais recourent très largement à un petit nombre d'acteurs spécialisés (agences immobilières, sociétés de relocation, bailleurs, diffuseurs d'informations) qui tendent à construire une offre locative répondant à un petit nombre de critères convergents : sécurité et prestige du quartier, accessibilité de certains lieux clés (l'École japonaise, les commerces japonais, la médiathèque de la Maison de la culture du Japon), proximité avec les individus de catégorie comparable, telle que les mères de famille au foyer ayant suivi leur mari en poste à l'étranger. En outre, les caractéristiques du bâti résidentiel parisien dans ces quartiers constituent une condition nécessaire à la concentration d'une population hétérogène du point de vue des ressources économiques. En effet, les appartements bourgeois datant de la seconde moitié du XIX siècle, qui composent majoritairement le secteur de Passy (XVI ${ }^{\mathrm{e}}$ ), par exemple, ont pour particularité d'associer aux grands apparte- ments des étages "nobles" des appartements et des chambres de taille réduite, historiquement réservés à la domesticité. Après rénovation et aménagement, ils peuvent satisfaire une clientèle d'étudiants célibataires, notamment. C'est ce qu'illustre la stratégie immobilière d'un propriétaire français qui met en location auprès des seuls étudiants japonais six "chambres de bonne" de taille réduite (10 à $15 \mathrm{~m}^{2}$ ) mais rénovées très soigneusement et qui bénéficient d'une localisation prestigieuse à Saint-Germain-des-Prés (VIe) et dans le Quartier latin $\left(V^{\mathrm{e}}\right)$.

Cependant, la carte des localisations résidentielles fait apparaître une distribution relativement large dans Paris intra-muros, à côté des secteurs de concentration évoqués précédemment. Cela s'explique par les parcours résidentiels des migrants japonais : si la plupart s'installent initialement dans le Centre-Ouest parisien, en recourant aux structures dédiées de l'accès au logement, seuls les expatriés en entreprise s'y maintiennent pendant les quelques années que dure leur séjour. 
Les autres connaissent généralement une mobilité résidentielle parfois très intense, au gré des évolutions du projet migratoire (par exemple, prolonger un séjour étudiant en optant pour une colocation plutôt que pour une chambre individuelle plus coûteuse), des opportunités
La carte de la distribution résidentielle des Japonais dans l'espace parisien montre clairement le poids de Paris (6o \%) et, en deuxième lieu, celui des Hauts-de-Seine (20\%) parmi l'ensemble des immigrés japonais dans l'espace francilien, qui concentre $70 \%$ des immigrés japonais. professionnelles, du souhait d'accéderàla propriété( $25 \%$ des immigrés japonais) ou encore des changements de situation matrimoniale.

L'installation en couple, et plus encore la naissance d'un enfant, déterminent très classiquement la mobilité et les choix résidentiels. Ces parcours sont souvent marqués par une sortie du segment dédié du parc locatif et par une diversification des lieux de résidence, ce qui s'explique par un processus de familiarisation avec l'espace parisien et son marché du logement.

En ce sens, on ne peut pas parler d'une stricte centralité japonaise d'un point de vue résidentiel. La question de la contribution des migrants japonais à la construction des territoires parisiens se pose donc plutôt dans le registre des pratiques non résidentielles.

\section{Une contribution paradoxale à la production des lieux parisiens}

Laquestion classique dela contribution des migrants internationaux aux dynamiques urbaines a fait l'objet de développements récents dans le champ de la recherche francophone, notamment à partir de la notion de "visibilité"?. Il s'agit de considérer l'ensemble des marquages symboliques et sociaux que produisent localement tel ou tel groupe de migrants du point de vue des autres citadins. Si cette approche pose d'épineux problèmes méthodologiques, car elle suppose d'appréhender finement une série de représentations par définition délicates à objectiver ${ }^{8}$, elle permet de considérer les migrants comme des "agents de la vie urbaine" à part entière.

Dans les secteurs valorisés de l'Ouest parisien, la présence visible de ménages japonais ajoute une dimension internationale à certaines scènes très locales, telles que les points de ramassage pour l'École franco-japonaise près du Trocadéro ou certaines rues commerçantes de Passy et de Beaugrenelle. Les agences immobilières utilisent d'ailleurs fréquemment la présence de Japonais à proximité comme un "label" de qualité à côté d'autres atouts du logement. C'est ce qu'illustrent ces extraits d'annonces affichées sur le site Internet d'une agence spécialisée dans les logements du Frontde-Seine : Very commercial and safe area, Japanese families residential area [...]" ; "Cave ; plusieurs familles japonaises dans la résidence ; possibilité de cuisine équipée (avec équipement neuf)". Ces quelques exemples montrent combien, au regard des professionnels du logement et, partant, des ménages résidant dans le voisinage, la présence de migrants internationaux associés à une position sociale élevée peut constituer un marqueur sociospatial très valorisant. On retrouve ici des observations faites dans d'autres contextes résidentiels de l'Ouest francilien où s'opère, dans les représentations communes des résidents comme des acteurs locaux, une claire distinction entre les "immigrés" d'une part et, d'autre part, les "expatriés" ressortissants de pays riches qui dessinent un environnement résidentiel "international" ou "cosmopolite" très positivement connoté ${ }^{10}$.

En dehors de ces secteurs de concentration résidentielle relative, la visibilité beaucoup plus ponctuelle des migrants japonais rend délicate l'identification de leur contribution spécifique aux marquages 
sociaux. On peut retenir toutefois le cas particulier des 500 à 1000 artistes et professionnels de la création qui bénéficient du fort attrait qu'exerce auprès du public parisien la culture japonaise au sens large, incluant aussi bien ses formes traditionnelles que les déclinaisons contemporaines de la pop culture. Les artistes japonais (musiciens, plasticiens, professeurs de danse, etc.) contribuent à conférer une dimension internationale à la quasi-totalité des scènes artistiques parisiennes ${ }^{11}$. Hormis ces cas de figure où la mise en scène d'une référence culturelle japonaise est constitutive de l'activité professionnelle, la faiblesse numérique des migrants et la rareté des équipements commerciaux ou culturels spécifiquement japonais ne donnent pas lieu à une identification collective de la part des autres usagers. Un bon exemple en est donné par le sentiment, maintes fois relaté dans les entretiens avec des Japonais résidant dans le haut Belleville notamment, d'être "pris pour des Chinois".

Les pratiques quotidiennes autour du logement ne constituent donc pas le principal vecteur de visibilité, qui relève plutôt de la concentration commerciale très singulière associée à la migration japonaise à Paris.

\section{Une centralité commerciale majeure : le quartier de l'Opéra}

Si la notion de "quartier" renvoie usuellement à un territoire fluctuant en termes de délimitation et de signification sociale ${ }^{12}$, il est notable que la dénomination de "quartier de l'Opéra" désigne une réalité urbaine identifiable de façon quasi constante dans les entretiens avec les migrants japonais et que, symétriquement, la concentration de restaurants japonais est presque systématiquement associée à ce quartier dans les guides et les blogs ${ }^{13}$. La plupart des commerces et restaurants japonais de la capitale sont concentrés dans un secteur hyper-central d'une vingtaine d'hectares structuré par la rue Sainte-Anne, à proximité de pôles culturels et touristiques (Opéra Garnier, Palais-Royal), commerciaux (Grands Magasins) et économiques (quartier de la Bourse) majeurs de l'espace parisien. À ce titre, le quartier de l'Opéra se distingue nettement du modèle des quartiers immigrés, qui se développent généralement dans des secteurs bénéficiant - au moins au départ - d'une faible attractivité auprès d'autres acteurs économiques ${ }^{14}$. La singularité urbaine du quartier japonais s'explique par son histoire, directement liée dès les années 1960 à la croissance rapide du nombre de touristes japonais effectuant des séjours à Paris ${ }^{15}$. En effet, les premiers établissements japonais sont des compagnies aériennes et des voyagistes, ainsi que des hôtels et des restaurants bon marché qui recherchent une situation proche des hauts lieux du tourisme à Paris. Puis s'im-
La plupart des commerces et restaurants japonais de la capitale sont concentrés dans un secteur hyper-central d'une vingtaine d'hectares. plantent des sociétés japonaises en lien avec les spécialisations économiques historiques du quartier : tourisme, commerce, banque, médias et industrie. Très rapidement, le quartier polarise donc les pratiques d'une très grande diversité de Japonais : touristes, résidents plus ou moins temporaires dans le quartier, à Paris ou dans d'autres villes européennes. Ces fréquentations renforcent localement une visibilité japonaise qui passe d'abord par l'offre commerciale, notamment en matière de restauration, via des vitrines et devantures souvent très marquées par des signes immédiatement reconnaissables, ou plus discrètement intégrées à un décor familier. 
Un exemple de la visibilité japonaise dans les commerces du quartier de l'Opéra. (c) HadRIEN Dubucs

\section{Les ambivalences du regard politique local}

Au regard des élus locaux, la question de l'emprise commerciale japonaise dans de "petits arrondissements" apparaît comme un enjeu politique de plus en plus significatif: "Il ne faudrait pas que la monoactivité de ce type de restauration fasse tache d'huile. Là, ça va, on a notre bonheur. On a ce qu'il faut pour manger japonais. Mais il ne faudrait pas plus. (...) Nous, on veut savoir jusqu'où ils vont aller, pourquoi ils sont concentrés là, comment on va faire. Et quel sera le visage de ce quartier dans dix ans, dans vingt ans. (...). Je le répète pour la énième fois, pas d’animosité particulière. Ce n'est pas un problème. Mais c'est quelque chose dont on commence à entendre parler, et c'est assez nouveau. Je n'en parlais pas il y a six ou sept ans. Mais depuis un ou deux ans, il n'y a pas un conseil de quartier de Palais-Royal, ou de Gaillon ou du Mail côté II', où on n'évoque pas cette question. (...) 'Ah, la boulangerie a fermé, la pharmacie aussi!' Pas grand-chose, mais quand même des pétitions. 150, 200, à l'échelle de notre arrondissement, c'est vraiment significatif' (entretien avec un membre du cabinet du maire du Ier arrondissement, juillet 2009). 
Le discours politique de qualification d'un tel phénomène est assez comparable à celui qu'observent les chercheurs sur d'autres quartiers concernés par une activité commerciale immigrée, tels que le quartier Sedaine-Popincourt $\left(\mathrm{XI}^{\mathrm{e}}\right)$, qui concentre des commerces textiles tenus par des Chinois ${ }^{16}$. Néanmoins, l'accent est plus mis sur la méconnaissance du phénomène que sur la réalité d'un "problème" économique. De plus, les chiffres de l'Insee concernant le nombre de résidents japonais (114 pour le $\mathrm{I}^{\text {er }}$ arrondissement, 88 pour le II en 1999) sont très clairement considérés comme sous-évalués par les acteurs politiques locaux. De tels écarts dans l'évaluation de la population japonaise peuvent s'expliquer par le fait qu'avec $3 \%$ de résidents étrangers, le quartier de l'Opéra est l'un des secteurs parisiens pour lesquels cette proportion est la plus faible ${ }^{17}$. Localement, les Japonais peuvent ainsi apparaitre comme l'une des seules populations étrangères, en particulier au sein du système scolaire: "On a une très grosse école privée à 100 mètres du quartier dont on parle. Je peux vous dire, mon fils y est inscrit en CM1. Une école qui accueille 426 gamins. École privée, catholique, donc sur le papier on ne penserait pas voir des Japonais! Depuis quelques années on voit arriver des enfants de parents japonais, et pareil, aucun problème, vraiment. Intégration parfaite, et culturellement, et scolairement. Alors, évidemment, ils ne vont pas au cathé, mais ça n'a aucune importance. Pas de problème particulier, pas de redoublement plus que la moyenne, c'est un peu une énigme, pour nous" (ibid.).

Ces propos suggèrent bien que les élus locaux perçoivent les résidents japonais comme une communauté "modèle" au regard de critères renvoyant à l'intégration" scolaire et culturelle. Dès lors, la visibilité japonaise apparaît à la fois très relative (car directement liée à la faiblesse numérique des habitants et plus encore des immigrés résidant dans l'arrondissement) et difficilement appréhendable par le politique.

\section{Un "quartier japonais" : les limites d'une dénomination convenue}

Quantitativement marginaux parmi les résidents, les Japonais sont également minoritaires parmi les usagers du quartier et, de plus en plus, parmi les commerçants ${ }^{18}$. Du point de vue des établissements commerciaux d'abord, les entrepreneurs japonais sont de plus en plus concurrencés par l'ouverture en nombre croissant de restaurants servant des plats japonais (sushis notamment) mais dont ni les patrons ni les cuisiniers ne sont japonais. Nourrie par le succès spectaculaire de la restauration japonaise à Paris (environ $+10 \%$ par an depuis la fin des années 1990 selon plusieurs études), cette offre commerciale soulève également l'épineuse question de l'"authenticité" gastronomique qui anime à la fois les guides ou blogs spécialisés et les acteurs institutionnels du commerce extérieur japonais. En témoigne, en 2007, la tentative - qui a fait long feu - de l'Office japonais du commerce extérieur d'instaurer pour la France un label "cuisine authentique" délivré par un Comité d'évaluation de la cuisine japonaise. Il est Au regard des élus locaux, la question de l'emprise commerciale japonaise dans de "petits arrondissements" apparaît comme un enjeu politique de plus en plus significatif.

les enquêtés japonais, rares sont ceux qui expriment une quelconque réticence à l'idée de fréquenter un restaurant japonais au seul motif qu'il serait tenu par un "Chinois", par exemple. À cela s'ajoute le nombre croissant de restaurants et de commerces alimentaires coréens dans - et autour de - la rue Sainte-Anne, ce qui contribue à complexifier l'éventail des cultures alimentaires consommables localement. En outre, l'analyse des usages du quartier et 
des pratiques d'approvisionnement oblige à nuancer doublement l'idée d'une spécialisation commerciale univoque associant étroitement clients et entrepreneurs japonais. Premièrement, les clients des commerces et restaurants japonais sont majoritairement des non-Japonais. À l'heure du déjeuner, pendant la semaine, il s'agit surtout de visiteurs et de salariés des sociétés alentour pour qui la présence visible de clients japonais est perçue comme un gage de qualité et d'authenticité. Deuxièmement, les usagers japonais du quartier ne recourent pas exclusivement à l'offre japonaise, loin s'en faut. Pour nombre de touristes japonais, par exemple, la rue Sainte-Anne n'est souvent qu'un itinéraire pittoresque et commode entre les grands magasins au nord et les attractions commerciales et touristiques de la rue de Rivoli, en bordure de Seine.

\section{Conclusion}

Parmi les pôles majeurs de l'immigration japonaise en Europe, Paris se singularise par la grande diversité des profils socioprofessionnels des Japonais qui y séjournent. Les cadres expatriés y côtoient des étudiants et des actifs aux parcours parfois aventureux, notamment dans le domaine de l'art. À cet égard, le cas des migrants japonais à Paris illustre parfaitement la complexification des mobilités internationales liées à un marché de la formation et de l'emploi inter-métropolitain. Socialement fragmentée en une multitude de "petits mondes" à base professionnelle, la population japonaise de Paris partage pourtant une territorialisation relativement cohérente. Du point de vue résidentiel d'abord, avec une relative concentration dans le centre-ouest de Paris, qui s'explique surtout par le rôle d'acteurs économiques et de vecteurs d'information japonais spécifiquement dédiés à l'accès au logement. Du point de vue des pratiques quotidiennes ensuite, avec une polarisation de celles-ci par le quartier de l'Opéra et son offre commerciale japonaise, dont le dyna- misme économique croissant soulève des enjeux urbains inédits : il s'agit pour les acteurs politiques locaux d'appréhender une concentration commerciale minoritaire qui, à la fois, cristallise un certain mécontentement de la part des riverains et contribue incontestablement à un dynamisme touristique et entrepreneurial qui dépasse très largement les clientèles et les commerces japonais. Peut-on encore parler d'un "quartier japonais" dès lors que les Japonais ne représentent qu'une part marginale des habitants et une minorité d'usagers et de commerçants ? Le terme de "visibilité" japonaise apparaît le plus approprié pour saisir les effets de décalage et de recomposition entre les représentations dominantes d'un lieu, auxquelles la figure de l'étranger apporte classiquement une contribution majeure, et la réalité des processus sociaux et économiques qui s'y jouent. 